\title{
Entropy generation analysis of Hall current effect on MHD micropolar fluid flow with rotation effect
}

\author{
Abiodun A. Opanuga ${ }^{1}$ (D) Olasunmbo O. Agboola ${ }^{1} \cdot$ Jacob A. Gbadeyan ${ }^{2} \cdot$ Hilary I. Okagbue $^{1}$
}

Received: 22 August 2019 / Accepted: 23 November 2019 / Published online: 4 December 2019

(c) Springer Nature Switzerland AG 2019

\begin{abstract}
Entropy generation in form of heat dissipation destroys useful energy which accounts for the underperformance and decrease in the thermodynamic efficiency of a system. Since micropolar fluid is used as a working fluid in many technological and industrial processes, it is therefore necessary to examine the influence of all factors that can enhance entropy production so as to achieve the best energy systems design. In this paper, the influence of Hall current and ion-slip on the entropy generation rate of micropolar fluid is investigated. An applied uniform magnetic field acts in a perpendicular direction to the flow of fluid. The nonlinear coupled partial differential equations used to model the micropolar fluid flow are transformed to ordinary differential equations by using appropriate similarity variables. The differential equations obtained are solved by applying differential transform method. The results for velocity profiles, temperature profile and microrotation are used to determine fluid irreversibility and Bejan number. To get a clearer view of the study, the effects of various parameters such as Hall, ion-slip, magnetic field and coupling number on primary velocity, secondary velocity, temperature profile, microrotation, entropy generation and Bejan number are presented and explained via plots. The results show that entropy generation is reduced as coupling number and magnetic parameter increase, Bejan number receives a boost with increase in coupling number and magnetic parameter. Furthermore, heat irreversibility is more dominant than fluid friction irreversibility at the region close to the channel walls.
\end{abstract}

Keywords Micropolar fluid · Entropy generation · Hall current · MHD · DTM

Mathematics Subject Classification 35J57 · 76D05 - 76W05

JEL Classification $\mathrm{C00} \cdot \mathrm{CO} 2$

List of symbols

$(u, w)$ Components of dimensional velocities

$(f, g) \quad$ Components of dimensionless velocities

$n^{2} \quad$ Micropolar parameter

$N \quad$ Coupling number

Gr Grashof number

Re Reynolds number

$G \quad$ Constant pressure gradient

$B_{0}^{2} \quad$ Uniform transverse magnetic field

$U_{0} \quad$ Characteristic velocity $a_{j} \quad$ Micro-inertial parameter

$C_{p} \quad$ Specific heat capacity

$R \quad$ Suction/injection Reynolds number

$h \quad$ Channel width

$k_{f} \quad$ Thermal conductivity

$m \quad$ Hall current parameter

M Hartmann number

Pr Prandtl number

$T \quad$ Temperature of fluid

$T_{0} \quad$ Reference temperature

$\triangle$ Abiodun A. Opanuga, abiodun.opanuga@covenantuniversity.edu.ng| ${ }^{1}$ Department of Mathematics, College of Science and Technology, Covenant University, Ota, Nigeria. ${ }^{2}$ Department of Mathematics, Faculty of Physical Science, University of Ilorin, Ilorin, Nigeria. 
$\mathrm{Br} \quad$ Brinkman number

$E_{G} \quad$ Local volumetric entropy generation rate

$K \quad$ Rotation parameter

Be Bejan number

Ns Dimensionless entropy generation parameter

\section{Greek letters}

$\rho \quad$ Fluid density

$\mu \quad$ Viscosity

$\sigma \quad$ Electrical conductivity

$\Omega \quad$ Temperature difference

$\Omega^{*} \quad$ Angular velocity

$\xi \quad$ Microrotation component

$\eta \quad$ Similarity variable

\section{Introduction}

Advancement in technology has led to a renewed interest in the investigation of non-Newtonian fluids because several important fluids available for engineering and industrial processes possess some flow properties that are not captured by Newtonian fluid model. As a result, various models have been proposed. Some of these are third grade fluid, couple stress fluid, micropolar fluid-a subclass of microfluids which was first investigated by Eringen [1]. Micropolar fluids have distinguishing features such as the local structure effects, which are microscopic and micro-motion of the fluid elements, stresses due to couple, and so on. It has important applications in exotic lubricants [2], animal blood [3], liquid crystals with rigid molecules [4], and some biological fluids [5]. Excellent research analysis on the significance and applications of this type of fluid has been investigated over the past few decades. Boundary layer flow solution of a polar fluid was considered by Ebert [6]. Heruska et al. [7] considered micropolar flow past a porous stretching sheet. Khonsari and Brew [8] presented the study on finite journal bearing with micropolar fluid used as a lubricant. Chemical reaction effect on micropolar fluid through vertical surface was studied by Gorla [9], Desseaux and Kelson [10] presented micropolar fluid flow through a stretching sheet. Nazar et al. [11] discussed the stagnation point flow of a micropolar fluid towards a stretching sheet.

In recent times, research work in this direction has attracted the attention of investigators such as Mahmoud and Waheed [12] who presented hydromagnetic flow and heat transfer of a micropolar fluid. Oahimire and Olajuwon [13] discussed the mixed convection hydromagnetic micropolar fluid. Perturbation technique was applied to tackle the dimensionless equations obtained from the governing equations. Olajuwon et al. [14] also investigated the unsteady case of viscoelastic mixed convection micropolar fluid with thermal radiation and Hall current effects.
Attention of several researchers has been drawn to the significance of Hall current and lon slip in the past few decades, due to the fact that several physical situations such as magnetohydrodynamic generators, Hall accelerators, flight magnetohydrodynamics, electrostatic precipitation require the inclusion of Hall current. It occurs in the presence of strong magnetic field or low density in an electrically conducting fluid.

In Hall current, the induced current in the fluid is conducted by electrons and collide vigorously with other neutral or charged particles. The collision of these charged particles resulted in the reduction of conductivity parallel to the electric field while it enhances the conductivity in the direction normal to both electric and magnetic fields.

Meanwhile studies have revealed that Coriolis force cannot be ignored due to its significant influence on fluid dynamics of the system. It induces secondary flow in the flow field. Its applications are found in the estimation of the flight path of rotating wheels and spin-stabilized missiles, monitoring the movement of oil and gas through the reservoirs, rotating heat exchangers, manufacturing of turbines and turbo mechanics. Further applications are in the studies of maintenance and secular variations in Earth's magnetic field due to motion of Earth's liquid core, internal rotation rate of the Sun, structure of the magnetic stars, solar and planetary dynamo problems, turbo machines, rotating MHD generators and rotating drum separators for liquid metal.

In view of the above, several investigations have been conducted. The first significant work on Hall-magnetohydrodynamic was carried out by Sato [15], the result shows that fluid flow becomes secondary in nature. Seth [16] presented the influence of Hall currents on unsteady hydromagnetic flow in a rotating channel with oscillating pressure gradient. Furthermore, Linga and Rao [17] considered the effect of Hall current on temperature distribution in a rotating ionized hydromagnetic flow between parallel walls. Bég et al. [18] studied the combined effects of Ohmic dissipation, Hall current and ionslip on transient MHD flow in a porous medium channel. Recently, Seth et al. [19] investigated the effects of Hall current and rotation on unsteady MHD couette flow in an inclined magnetic field. Very recently, Animasun et al. [20] investigated the significance of Lorentz force and thermoelectric on the flow of $29 \mathrm{~nm}$ cuo-water nanofluid on an upper horizontal surface of a paraboloid of revolution. It was posited that at higher values of Hall parameter the domain of cross-flow velocity can be significantly reduced. Other related studies are [21-25].

However, the irreversibility analysis of micropolar fluid has not been adequately considered despite the fact that the performance of thermal devices is always affected by fluid irreversibility which usually results in the enhancement of entropy generation and reduction of thermal efficiency. Since the introduction of second law of thermodynamics 
for irreversibility analysis by its proponent, Bejan [26], the approach has found application in several fluids flow such as third grade fluid [27-29], couple stress fluid [30-33], nanofluid [34], Couette flow [35], Poiseuille flow [36] and even micropolar fluid [37, 38], others are found in Refs. $[39,40]$. Motivated by Srinivasacharya and Bindu [38], this work seeks to analyse the entropy generation due to Hallmagnetohydrodynamics and Coriolis effects on micropolar fluid flow. The solutions of the governing equations are obtained by an efficient and rapidly convergent differential transform method (DTM). The convergence of this technique is addressed in the following references [41-43].

\subsection{Mathematics analysis}

A steady magnetohydrodynamic incompressible, viscous and electrically conducting micropolar fluid between two infinite vertical plates of distance $2 h$ is investigated. The coordinate system chosen is such that the $x$-axis is along the vertical upward direction, the $y$-axis is along the width of the plate and the $z$-axis is taken normal to the plane of the plate, as depicted in Fig. 1. Assumption that the physical quantities depend on $y$ only is also taken. In addition, a magnetic field of constant strength $B_{0}$ is introduced in a direction parallel to $z$-axis in a perpendicular direction to the fluid flow. The effect of fluid polarization is not significant due to the assumption that applied or polarized voltage is not introduced corresponding to case where there is no addition or removal of energy from the fluid by any electrical means. It is further assumed that the entire system rotates about the normal axis to the plate with an angular velocity of $\Omega^{*}$ and the plate is subjected to a constant suction such that $v_{0}>0$ is the velocity for suction and $v_{0}<0$ is the velocity of injection. The equations governing the flow of fluid under the usual Boussinesq approximation are given by

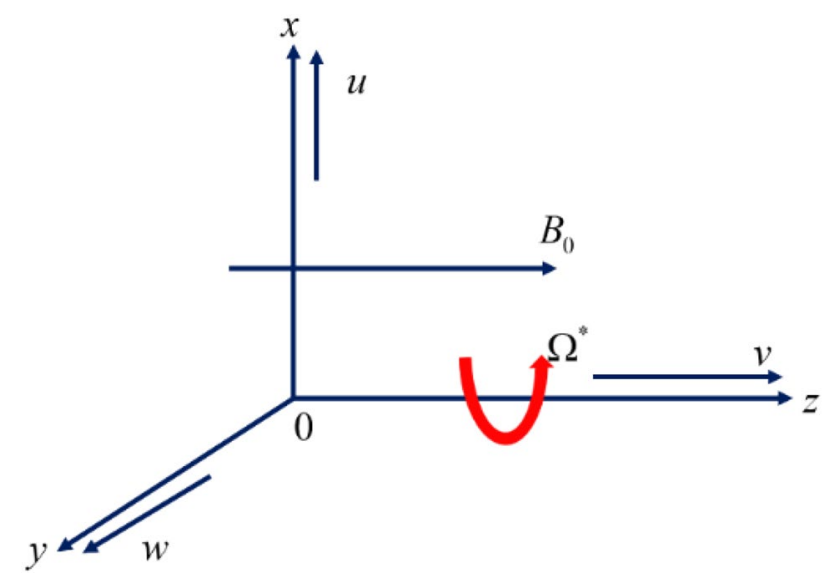

Fig. 1 Diagrammatic representation of the problem

\section{Continuity equation}

$\frac{\partial v}{\partial y}=0$

\section{Momentum equation in $x$-direction}

$$
\begin{gathered}
(\mu+\kappa) \frac{\partial^{2} u}{\partial y^{2}}-\rho v_{0} \frac{\partial u}{\partial y}-2 \Omega^{*} w+\kappa \frac{\partial \xi}{\partial y}+\rho g \beta\left(T-T_{1}\right) \\
+\frac{\sigma B_{0}^{2}}{\left(1+m^{2}\right)}(u+m w)-\frac{\partial p}{\partial x}=0
\end{gathered}
$$

\section{Momentum equation along $y$-direction}

$$
(\mu+\kappa) \frac{\partial^{2} w}{\partial y^{2}}-\rho v_{0} \frac{\partial w}{\partial y}+2 \Omega^{*} u-\kappa \frac{\partial \xi}{\partial y}-\frac{\sigma B_{0}^{2}}{\left(1+m^{2}\right)}(m u-w)=0
$$

\section{Angular momentum equation}

$\gamma \frac{\partial^{2} \xi}{\partial y^{2}}-\rho j^{*} v_{0} \frac{\partial N}{\partial y}-2 \kappa \xi-\kappa \frac{\partial u}{\partial y}=0$

\section{Energy equation}

$$
\begin{aligned}
& k_{f} \frac{\partial^{2} T}{\partial y^{2}}-\rho c_{p} v_{0} \frac{\partial T}{\partial y}+(\mu+\kappa)\left[\left(\frac{\partial u}{\partial y}\right)^{2}+\left(\frac{\partial w}{\partial y}\right)^{2}\right] \\
& \quad+2 \kappa\left(\xi^{2}+\xi \frac{\partial u}{\partial y}\right)+\gamma\left(\frac{\partial \xi}{\partial y}\right)^{2}+\sigma B_{0}^{2}\left(u^{2}+w^{2}\right)=0
\end{aligned}
$$

and the boundary conditions:

$u(h)=w(h)=\xi(h)=0, \quad T^{\prime}(h)=\frac{q}{k_{f}}$,
$u(-h)=w(-h)=\xi(-h)=0, \quad T(-h)=T_{1}$

The following dimensionless variables are introduced

$\eta=\frac{y}{d}, \quad u=U_{0} f, \quad w=U_{0} g, \quad \xi=\frac{U_{0}}{d} h, \quad \theta(\eta)=\frac{T-T_{1}}{\frac{q d}{k_{f}}}$,

$N=\frac{\kappa}{\kappa+\mu}, \operatorname{Pr}=\frac{\rho C_{p}}{k_{f}}, \operatorname{Re}=\frac{\rho U_{0} d}{\mu}$,

$R=\frac{\rho v_{0} d}{\mu}, \operatorname{Gr}=\frac{\rho^{2} g \beta q d^{4}}{\mu^{2} k_{f}}, M^{2}=\frac{\sigma B_{0}^{2} d^{2}}{\mu}$,

$A=\frac{d^{2}}{\mu U_{0}} \frac{\partial p}{\partial x}, \quad a_{j}=\frac{j^{*}}{d^{2}}, \quad n^{2}=\frac{d^{2} \kappa(2 \mu+\kappa)}{\gamma(\mu+\kappa)}, B r=\frac{\mu U_{0}^{2}}{k_{f}\left(T_{2}-T_{1}\right)}$

It is obvious that the continuity Eq. (1) is satisfied by using (7) and (2)-(6) yield

$$
\begin{gathered}
\frac{1}{1-N} f^{\prime \prime}+R f^{\prime}+K^{2} g-\frac{N}{1-N} h^{\prime}+\frac{\mathrm{Gr}}{\operatorname{Re}} \theta-A \\
+\frac{M^{2}}{\left(1+m^{2}\right)}(f+m g)=0
\end{gathered}
$$


$\frac{1}{1-N} g^{\prime \prime}+R g^{\prime}-K^{2} f+\frac{N}{1-N} h^{\prime}+\frac{M^{2}}{\left(1+m^{2}\right)}(m f-g)=0$

$\frac{2-N}{n^{2}} h^{\prime \prime}-a_{i} R\left(\frac{1-N}{N}\right) h^{\prime}-2 h-f^{\prime}=0$
The inverse differential transform of $F(s)$ is given by

$$
f(y)=\sum_{s=0}^{\infty} y^{s} F(y)
$$

Some of the basic DTM theorems that are applicable in this paper are as follows:

$\theta^{\prime \prime}-\operatorname{Re} \operatorname{Pr} \theta^{\prime}+\frac{\operatorname{Br}}{1-N}\left[f^{\prime 2}+g^{\prime 2}+2 N\left(h^{2}+h f^{\prime}\right)+\frac{N(2-N)}{n^{2}} h^{\prime 2}+M^{2}\left(f^{2}+g^{2}\right)\right]=0$

$f=0, g=0, h=0, \theta^{\prime}=1 ; \eta=1: f=0$,

$g=0, \quad h=0, \quad \theta^{\prime}=0 ; \eta=-1$

In this work, primes are used to denote differentiation with respect to $\eta$.

\subsection{Differential transform solution procedure}

The basic principle of differential transformation method (DTM) is introduced in this section. Consider a function, $f(y)$, then the differential transform of the $k$ th derivative of $f(y)$ is given by
Theorem 1 If $f(y)=p(y) \pm q(y)$, then $F(s)=P(s) \pm Q(s)$.

Theorem 2 If $f(y)=\tau p(y)$, then $F(s)=\tau P(s)$, where $\tau$ is a given constant.

Theorem 3 If $f(y)=\frac{d^{n} p(y)}{d y^{n}}$, then $F(s)=(s+1)(s+2) \ldots$ $(s+n) P(s+n)$.

Theorem 4 If $f(y)=p(y) q(y)$, then $F(s)=\sum_{r=0}^{s} P(r) Q(s-r)$.

By applying DTM and relevant theorems from Theorems 1, 2, 3 and 4, one obtains the following iterative schemes:

$$
\begin{aligned}
F(s+2)= & -\frac{1-N}{(s+1)(s+2)}\left[R(s+1) F(s)+K^{2} G(s)-\frac{N}{1-N}(s+1)+\frac{G r}{\operatorname{Re}} \Theta(s)-A+\frac{G r}{\operatorname{Re}} \Theta(s)+\frac{M^{2}}{\left(1+m^{2}\right)}\{F(s)+\right. \\
G(s+2)= & -\frac{1-N}{(s+1)(s+2)}\left[R(s+1) G(s+1)-K^{2} F(s)+\frac{N}{1-N}(s+1) H(s)+\frac{M^{2}}{\left(1+m^{2}\right)}\{m F(k)-G(s)\}\right] \\
H(s+2)= & \frac{n^{2}}{(2-N)(s+2) !}\left[a_{i} R\left(\frac{1-N}{N}\right)(s+1) H(s+1)+2 H(s)+(s+1) F(s+1)\right] \\
\Theta(s+2)= & \frac{1}{(s+1)(s+2)}\left[\operatorname{RePr}(s+1) \Theta(s+1)-\frac{B r}{1-N}\left\{\sum_{r=0}^{s}(r+1)(s-r+1) F(r+1) F(s-r+1)\right.\right. \\
& +\sum_{r=0}^{s}(r+1)(s-r+1) G(r+1) G(s-r+1)+2 N\left(H(r) H(s-r)+\sum_{r=0}^{s} H(r)(s-r+1) F(s-r+1)\right) \\
& \left.\left.+\frac{N(2-N)}{n^{2}} \sum_{r=0}^{s}(r+1)(s-r+1) H(r+1) H(s-r+1)+M^{2}(F(r) F(s-r)+G(r) G(s-r))\right\}\right]
\end{aligned}
$$

$F(s)=\frac{1}{s !}\left[\frac{d^{s} f(y)}{d y^{s}}\right]_{y=0}$ where $F(s), G(s), H(s)$ and $\Theta(s)$ are the differential transformed functions of $f(\eta), g(\eta), h(\eta)$ and $\theta(\eta)$ respectively. 
Let us designate

$F(0)=a_{1}, F(1)=a_{2}$,

$G(0)=b_{1}, G(1)=b_{2}$,

$H(0)=c_{1}, H(1)=c_{2}$,

$\Theta(0)=d_{1} \Theta(1)=d_{2}$,

whose values are to be determined using $f=g=h=0, \theta^{\prime}=1, \quad \eta=1$ and $f=g=h=\theta^{\prime}=0, \quad \eta=-1$.

The approximants for $f(\eta), g(\eta), h(\eta)$ and $\theta(\eta)$ can now be obtained using the iterative formulas in Eqs. (15)-(18).

The entropy generation expression for an incompressible micropolar fluid can be stated as [26]
The Bejan number ratio in Eq. (22) is an indication of Bejan number variation from 0 to 1 which reveals that viscous dissipation irreversibility is dominant when $\mathrm{Be}=0$ and heat transfer irreversibility dominates entropy production at $\mathrm{Be}=1$. However, equal contribution from viscous dissipation irreversibility and heat transfer irreversibility to entropy production is noted when $\mathrm{Be}=0.5$.

To validate the results obtained using the differential transform technique described above, the results are compared with Srinivasacharya and Bindu [38] as shown in Table 1 at $N=0.1, n=1, A=-1, R=0, \alpha=0, K=0, G r=0$,

$E_{G}=\frac{k_{f}}{T_{1}^{2}}\left(\frac{\partial^{2} T}{\partial y^{2}}\right)^{2}+\frac{\mu+k}{T_{1}}\left[\left(\frac{\partial u}{\partial y}\right)^{2}+\left(\frac{\partial w}{\partial y}\right)^{2}\right]+\frac{2 k}{T_{1}}\left(\xi^{2}+\xi \frac{\partial u}{\partial y}\right)+\frac{\gamma}{T_{1}}\left(\frac{\partial \xi}{\partial y}\right)^{2}+\frac{\sigma B_{0}^{2}}{T_{1}}\left(u^{2}+w^{2}\right)=0$

and the dimensionless form is obtained by using (7) in

(20) to give

$N_{S}=\left(\theta^{\prime}\right)^{2}+\frac{B r}{1-N}\left[f^{\prime 2}+g^{\prime 2}+2 N\left(h^{2}+h f^{\prime}\right)+\frac{N(2-N)}{n^{2}} h^{\prime 2}+M^{2}\left(f^{2}+g^{2}\right)\right]$

The entropy generation distribution is determined by

Bejan number (Be), this is stated as

$\mathrm{Be}=\frac{N_{1}}{N_{s}}=\frac{1}{1+\Phi}, \quad \Phi=\frac{N_{2}}{N_{1}}$,

$M=0$ are presented. The approximate solutions for the velocity (8) and micrototation (10) profiles are obtained as

where

$N_{1}=\left(\frac{\partial \theta}{\partial \eta}\right)^{2}, N_{2}=\frac{B r}{1-N}\left[\left(\frac{\partial f}{\partial \eta}\right)^{2}+\left(\frac{\partial g}{\partial \eta}\right)^{2}+2 N\left(h^{2}+h f^{\prime}\right)+\frac{N(2-N)}{n^{2}} h^{\prime 2}+M^{2}\left(f^{2}+g^{2}\right)\right]$

Table 1 Comparison of the solution of the velocity and microtation profiles with Srinivasacharya and Bindu [38] at $N=0.1, n=1, A=-1, R=0$, $\alpha=0, K=0, \mathrm{Gr}=0, M=0$

\begin{tabular}{|c|c|c|c|c|}
\hline \multirow[t]{2}{*}{$\eta$} & \multicolumn{2}{|l|}{ Velocity profile $f(\eta)$} & \multicolumn{2}{|c|}{ Microrotation profile $h(\eta)$} \\
\hline & $\begin{array}{l}\text { Srinivasacharya and } \\
\text { Bindu [38] }\end{array}$ & Present & $\begin{array}{l}\text { Srinivasacharya and } \\
\text { Bindu [38] }\end{array}$ & Present \\
\hline-1 & 0 & $-6.9889 \times 10^{-18}$ & 0 & $-1.4374 \times 10^{-18}$ \\
\hline-0.8090 & 1.557 & 1.557 & -0.0204 & -0.0204 \\
\hline-0.6129 & 0.2817 & 0.2817 & -0.0275 & -0.0275 \\
\hline-0.4258 & 0.3696 & 0.3696 & -0.0248 & -0.0248 \\
\hline-0.2181 & 0.4302 & 0.4302 & -0.0147 & -0.0147 \\
\hline 0 & 0.4518 & 0.4518 & 0 & $-1 \times 10^{-18}$ \\
\hline 0.2181 & 0.4302 & 0.4302 & 0.0147 & 0.0147 \\
\hline 0.4258 & 0.3696 & 0.3696 & 0.0248 & 0.0248 \\
\hline 0.6129 & 0.2817 & 0.2817 & 0.0275 & 0.0275 \\
\hline 0.8090 & 1.557 & 1.557 & 0.0204 & 0.0204 \\
\hline 1 & 0 & $-6.9869 \times 10^{-18}$ & 0 & $-2.5645 \times 10^{-18}$ \\
\hline
\end{tabular}


Fig. 2 Velocity profiles for Hall curent (m), ion-slip (K), coupling number $(\mathrm{N})$ and magnetic field $(\mathrm{M})$ parameter
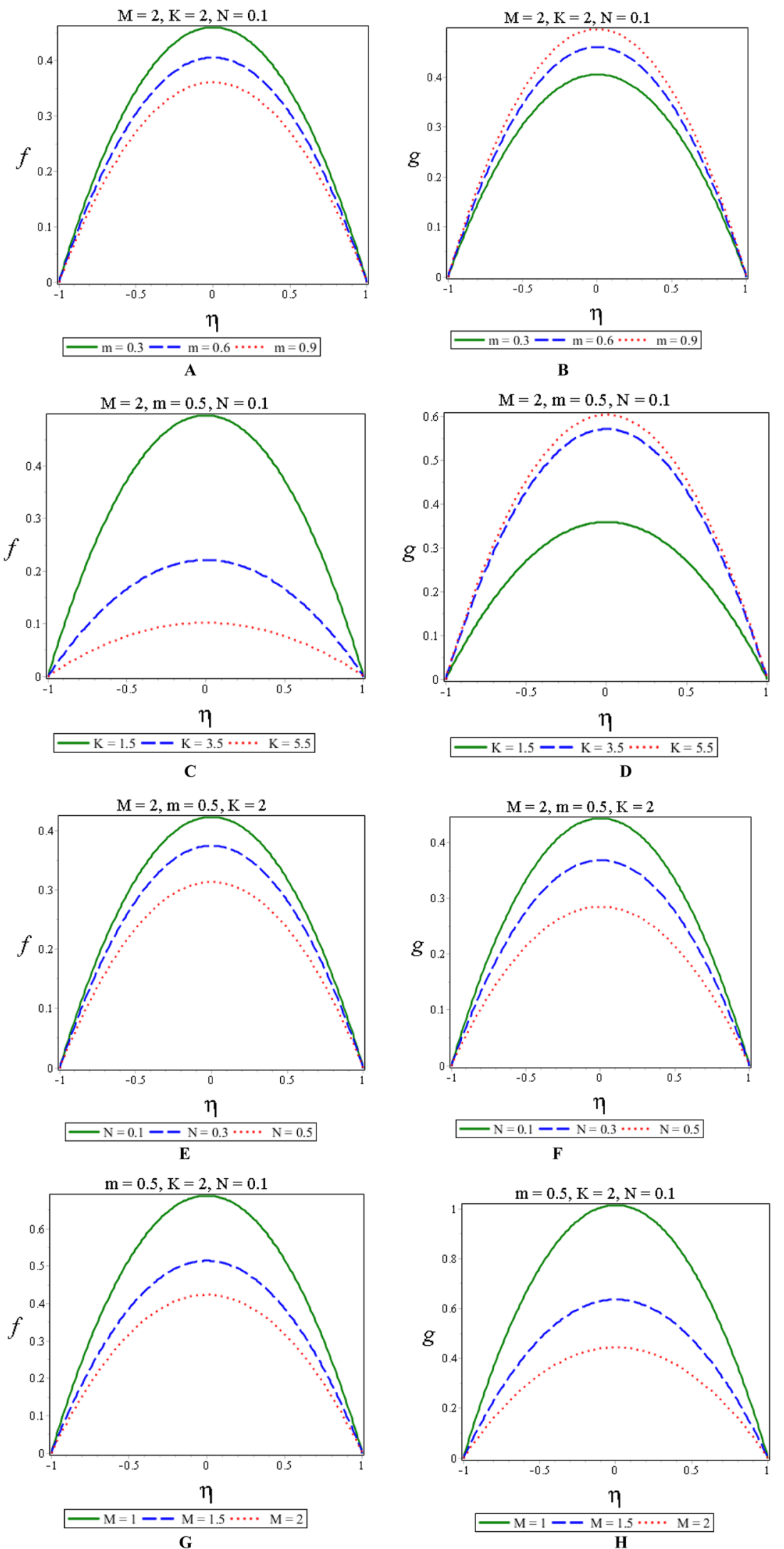

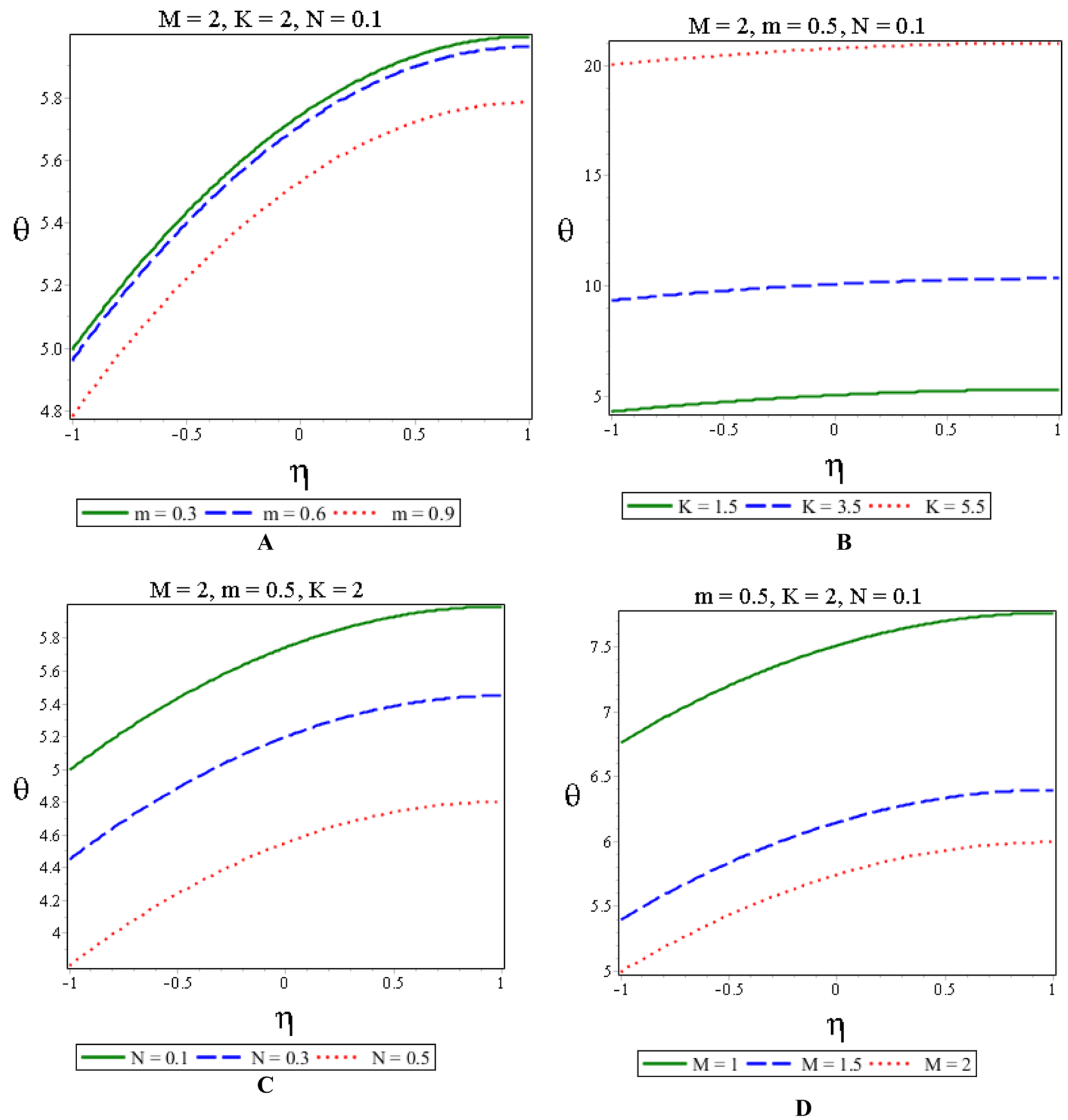

Fig. 3 Temperature profiles for Hall curent (m), ion-slip (K), coupling number $(N)$ and magnetic field parameter (M)

$$
\begin{aligned}
f(\eta)= & 0.45179445044303724-0.45353088643367002 \eta^{2}+1.7543859649122807 \times 10^{-20} \eta^{3} \\
& +0.0016794436743871474 \eta^{4}+8.7719298245614035 \times 10^{-22} \eta^{5}+0.000055981455812904913 \eta^{6} \\
& +2.0885547201336675 \times 10^{-23} \eta^{7}+9.9966885380187337 \times 10^{-7} \eta^{8}+2.9007704446300936 \times 10^{-25} \eta^{9}+\ldots
\end{aligned}
$$




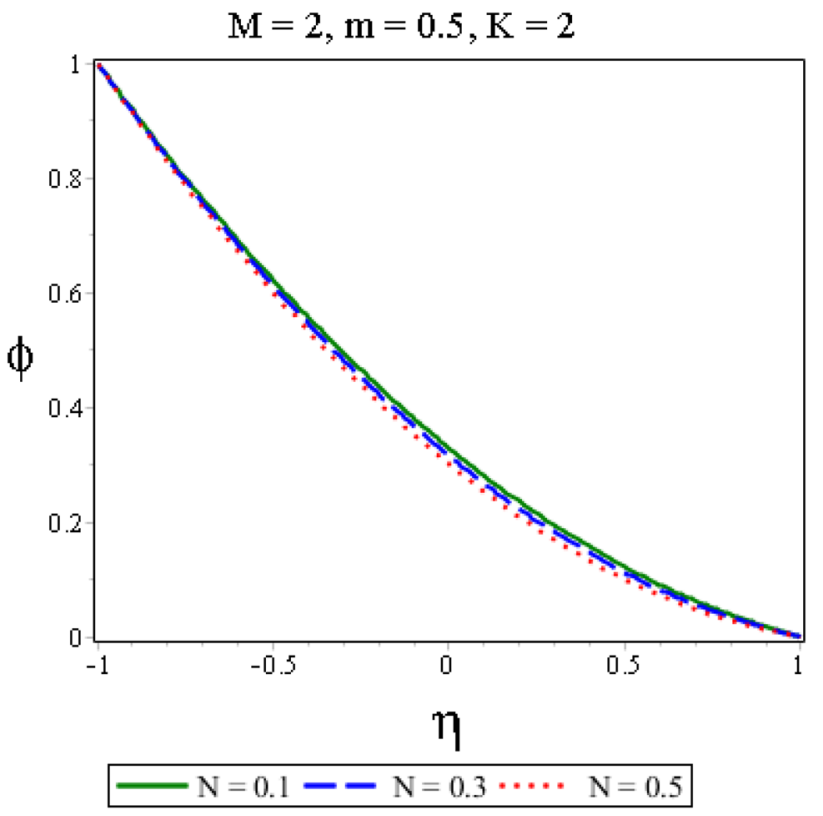

Fig. 4 Microrotation profile for coupling number (N)

and

$$
\begin{aligned}
h(\eta)= & -1 \times 10^{-18}+0.070617728673400420 \eta-5.2631578947368422 \times 10^{-19} \eta^{2} \\
& -0.057177746975485895 \eta^{3}-4.3859649122807017 \times 10^{-20} \eta^{4}-0.0033588873487742948 \eta^{5} \\
& -1.4619883040935672 \times 10^{21} \eta^{6}-0.000079973508304149875 \eta^{7}-2.606934001670843 \times 10^{-23} \eta^{8}
\end{aligned}
$$

\section{Results and discussion}

The equations governing the motion of a micropolar fluid as shown in Eqs. (7)-(11) are analytically tackled using differential transform technique. The effects of various physical parameters governing the flow on primary velocity, secondary velocity, temperature, microrotation, entropy generation and Bejan number are carried out for $1 \leq M \leq 2,0.1 \leq N \leq 0.5,1 \leq K \leq 6$ and $0.3 \leq m \leq 1$ while $n=2, A=-1, \mathrm{Gr}=1, s=1, a_{j}=0.001, \operatorname{Pr}=0.71, \mathrm{Br}=0.5$ are fixed.

In Fig. 2 the response of fluid velocity to variation in Hall current parameter, ion-slip parameter, coupling number and magnetic field parameter is displayed. In Fig. 2a, $b$ primary velocity decelerates and reached minimum at $\eta=0$ while secondary velocity is enhanced and attained maximum at $\eta=0$. The boost in secondary velocity is due to the reduction in the damping effect of magnetic field as the values of Hall parameter rise. This implies that higher values of Hall parameter reduce the resistive force imposed by the applied magnetic field. It is observed in
The effects of magnetic parameter and coupling number on the entropy generation and Bejan number are presented in Figs. 5 and 6. It is noted in Fig. 5 a that there is no significant change in entropy generation as Hall parameter values vary. However, reduction in entropy generation is observed as Ion-slip parameter, magnetic parameter and coupling number increase in magnitude as depicted in Fig. 5b, c, d. The result of this work could be utilised to scale down entropy generation rate in systems design and manufacturing industries. It further reveals that entropy generation minimisation (EGM) goal is realisable. Finally, in Fig. $6 a$, b the Bejan number is enhanced as magnetic field parameter and coupling number increase. However, the rise in Bejan number is more significant at the channel walls region, this indicates the dominance of heat transfer irreversibility over fluid friction irreversibility. Generally, it is observed that the plots for the entropy generation and Bejan number are parabolic and as $N \rightarrow 0$ the entropy generation is drastically reduced at the channel walls but becomes less pronounced at the centre line. 


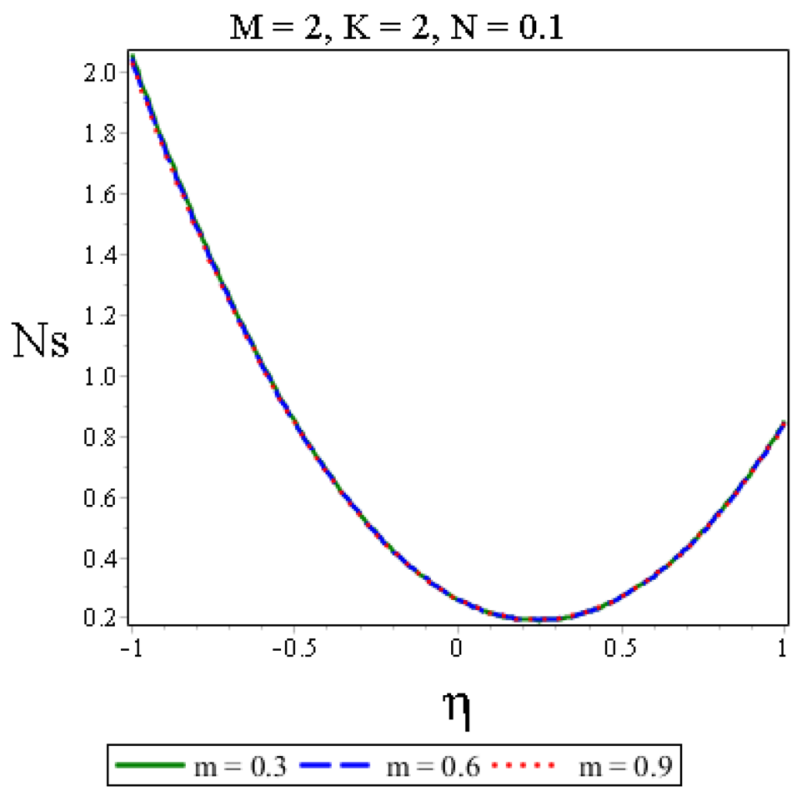

A

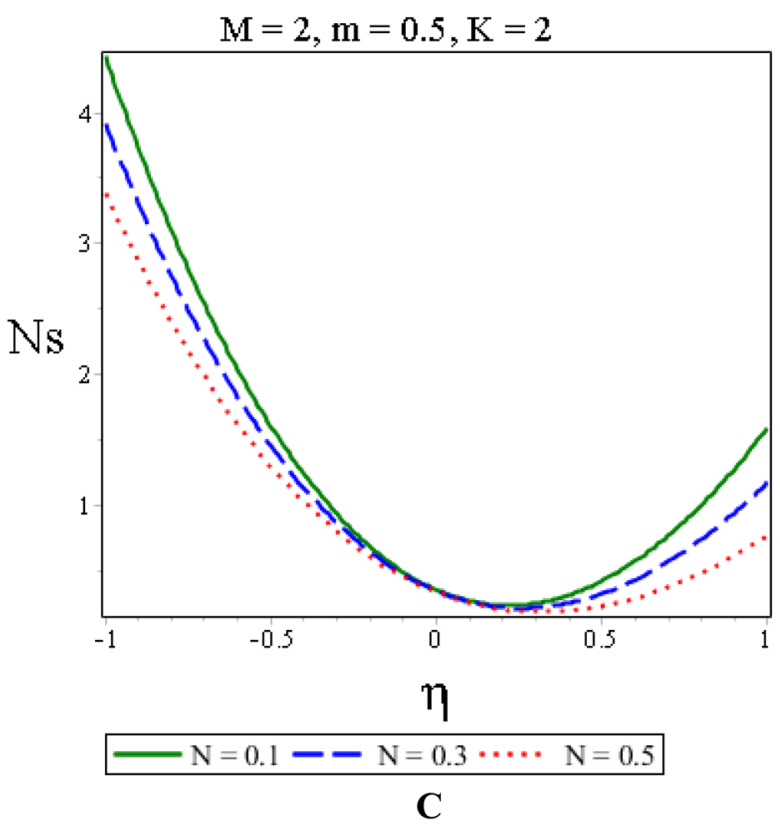

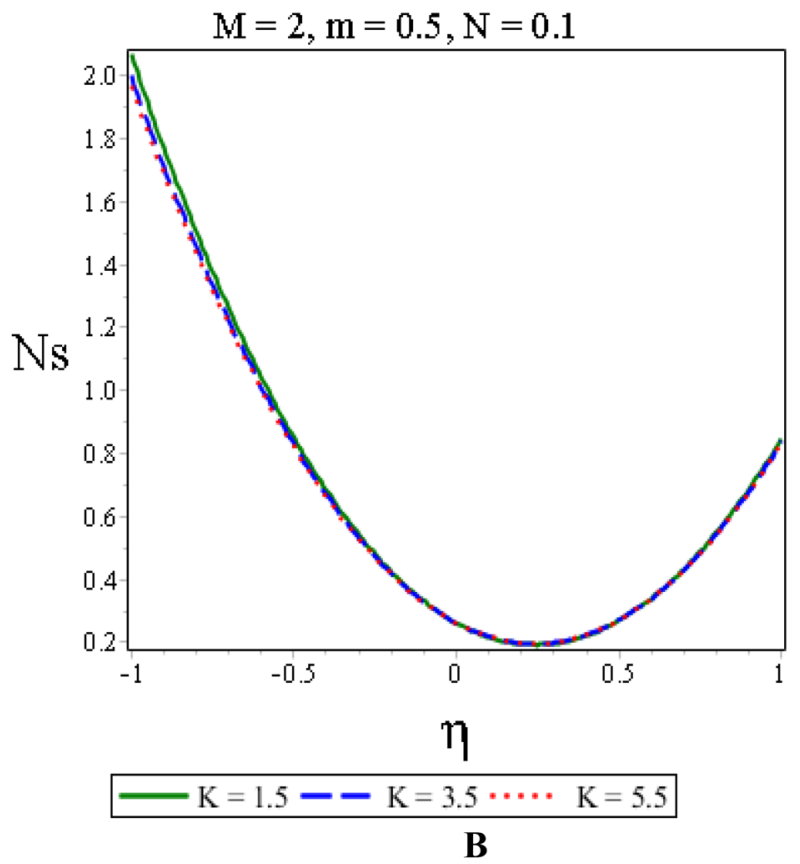

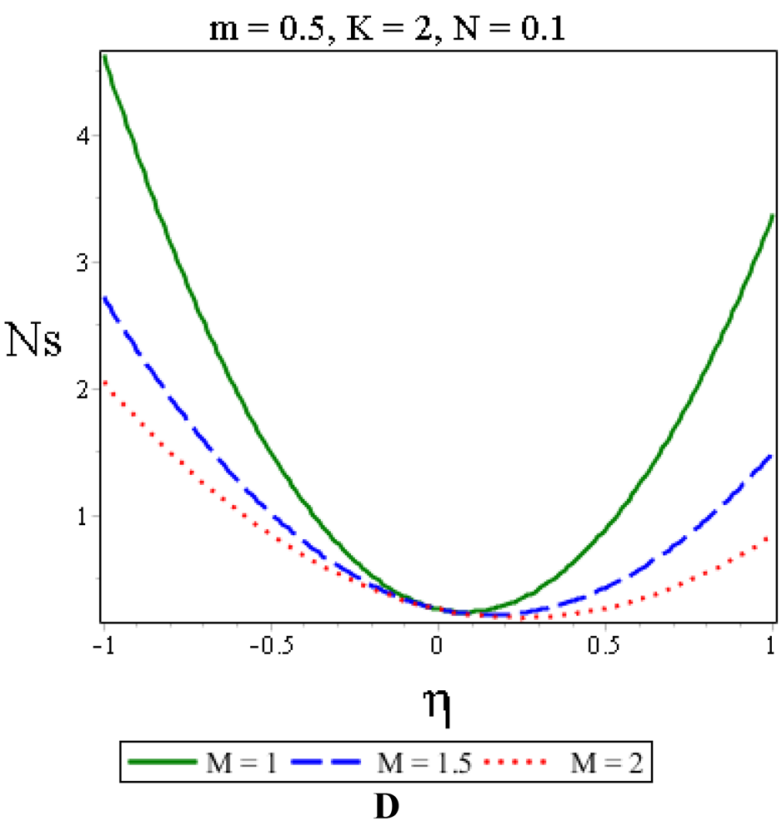

Fig. 5 Entropy generation for Hall curent (m), ion-slip (K), coupling number (N) and magnetic field parameter (M)

\section{Conclusion}

In this study, investigation has been conducted on the entropy generation of Hall and ion-slip effects on MHD micropolar flow past a vertical plate. The differential transform technique was employed to obtain the solutions of the velocity, temperature and microrotation profiles. The solutions are substituted in the expressions for the entropy generation and Bejan number. The results are presented and explained using Figs. 2, 3, 4, 5 and 6. The main findings of this present investigations are outlined below.

(i) Increase in Hall effect, ion-slip parameter, coupling number and magnetic parameter increase primary velocity;

(ii) Secondary velocity increases with Hall and ion-slip effects while coupling number and magnetic field reduce secondary velocity; 

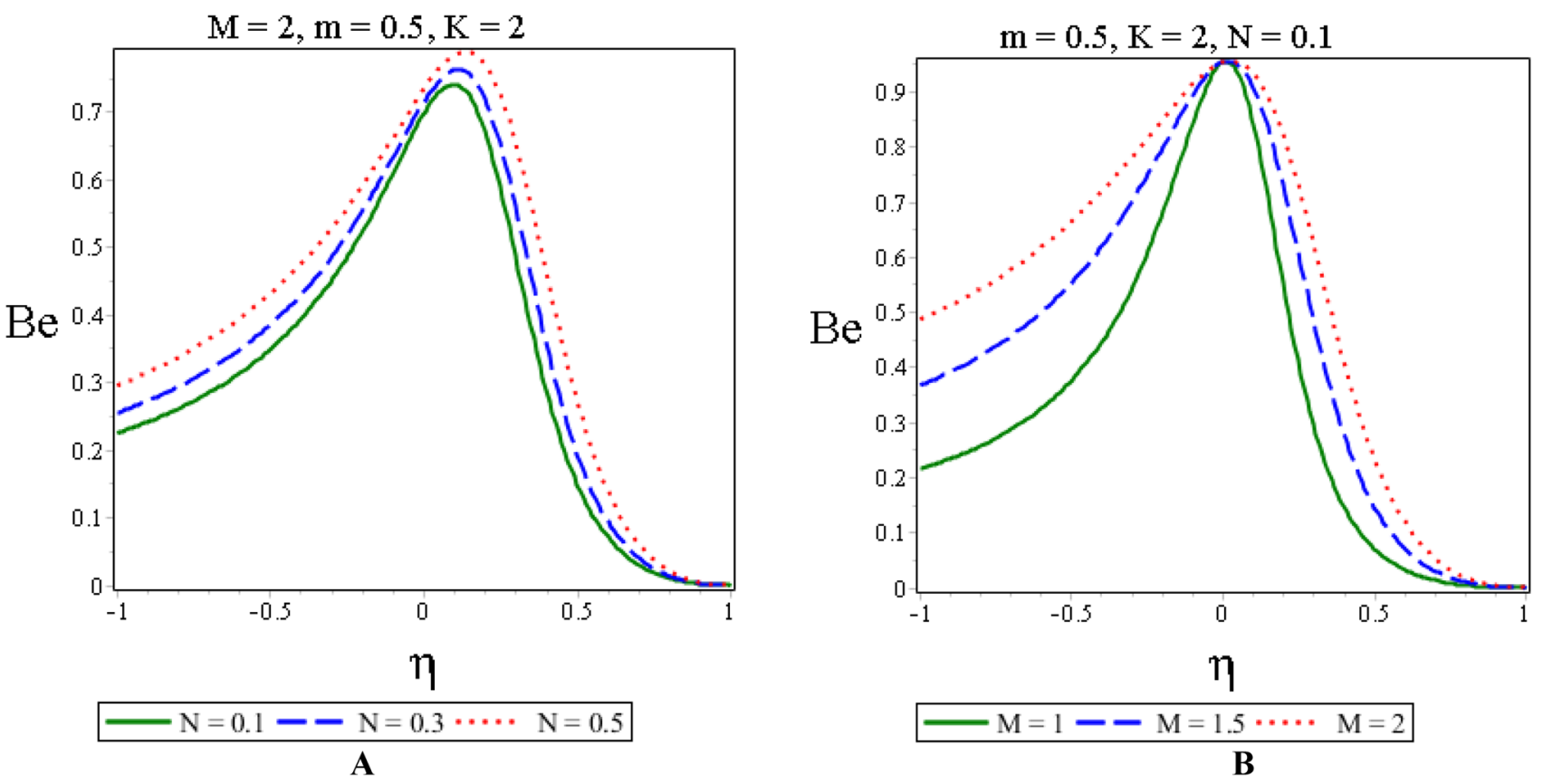

Fig. 6 Bejan number for magnetic parameter (M) and coupling number (N)

(iii) Temperature profile is reduced as Hal parameter, coupling number and magnetic parameters are increased while ion-slip e parameter increased fluid temperature;

(iv) Coupling number reduced microrotation;

(v) Entropy generation is reduced as coupling number and magnetic parameter are increased;

(vi) Bejan number is enhanced with increase in coupling number and magnetic parameter;

(vii) Heat irreversibility is more dominant than entropy generation due to fluid friction at the region close to the wall channels than at the centreline.

\section{Compliance with ethical standards}

Conflict of interest The authors declare that they have no competing interests.

\section{References}

1. Eringen AC (1966) Theory of micropolar fluids. J Math Mech 16:1-18

2. Eringen AC (2001) Microcontinuum field theories II: fluent media. Springer, New York

3. Ariman T, Turk MA, Sylvester ND (1974) Applications of microcontinunm fluid mechanics. Int J Eng Sci 12:273-293
4. Lockwood F, Benchaitra M, Friberg S (1987) Study of polytropic liquid crystals in viscometric flow and clastohydrodynamic contact. ASL Tribol Trans 30:539-548

5. Hadimoto B, Tikioka T (1969) Two dimensional shear flows of linear micropolar fluids. Int J Eng Sci 7:515-522

6. Ebert $F$ (1973) A similarity solution for the boundary layer flow of a polar fluid. J Chem Eng 5:85-92

7. Heruska MW, Watson LT, Sankara KK (1986) Micropolar flow past a porous stretching sheet. Comput Fluids 14:117-129

8. Khonsari MM, Brew D (1989) On the performance of finite journal bearing lubricated with micropolar fluids. ASLE Tribol Trans 32:155-160

9. Gorla RSR (1992) Mixed convection in a micropolar fluid from a vertical surface with uniform heat flux. Int J Eng Sci 30:349-358

10. Desseaux A, Kelson NA (2000) Flow of a micropolar fluid bounded by a stretching sheet. Anziam J 42:C536-C560

11. Nazar R, Amin N, Filip D, Pop I (2004) Stagnation point flow of a micropolar fluid towards a stretching sheet. Int J Non-Linear Mech 39(7):1227-1235

12. Mahmoud MAA, Waheed SE (2012) MHD flow and heat transfer of a micropolar fluid over a stretching surface with heat generation (absorption) and slip velocity. J Egypt Math Soc 20:20-27

13. Oahimire Jl, Olajuwon BI (2014) Effect of Hall current and thermal radiation on heat and mass transfer of a chemically reacting MHD flow of a micropolar fluid through a porous medium. J King Saud Univ Eng Sci 26:112-121

14. Olajuwon BI, Oahimire Jl, Ferdow M (2014) Effect of thermal radiation and Hall current on heat and mass transfer of unsteady MHD flow of a viscoelastic micropolar fluid through a porous medium. Eng Sci Technol Int J 17:185-193

15. Sato $H$ (1961) The Hall effect in the viscous flow of ionized gas between parallel plates under transverse magnetic field. J Phys Soc Jpn 16(7):1427-1435

16. Seth GS, Ghosh SK (1986) Effect of Hall currents on unsteady hydromagnetic flow in a rotating channel with oscillating pressure gradient. Indian J Pure Appl Math 17(6):819-826 
17. Linga RT, Ramana Rao VV (1993) Hall effects on temperature distribution in a rotating ionized hydromagnetic flow between parallel wall. Int J Eng Sci 31(7):1073-1091

18. Bég OA, Zueco J, Takhar HS (2009) Unsteady magnetohydrodynamic Hartmann-Couette flow and heat transfer in a Darcian channel with Hall current, ionslip, viscous and Joule heating effects: network numerical solutions. Commun Nonlinear Sci Numer Simul 14:1082-1097

19. Seth GS, Nandkeolyar R, Ansari MdS (2015) Effects of Hall current and rotation on unsteady MHD Couette flow in the presence of an inclined magnetic field. J Appl Fluid Mech 8(1):7-20

20. Animasaun IL, Mahanthesh B, Jagun AO, Bankole TD, Sivaraj $R$, Shah NA, Saleem S (2019) Significance of Lorentz force and thermoelectric on the flow of $29 \mathrm{~nm} \mathrm{CuO}$-water nanofluid on an upper horizontal surface of a paraboloid of revolution. J Heat Transf 141:022402. https://doi.org/10.1115/1.4041971

21. Opanuga AA, Bishop SA, Okagbue HI, Agboola OO (2018) Hall current and joule heating effects on flow of couple stress fluid with entropy generation. Eng Technol Appl Sci Res 8(3):2923-2930

22. Garg BP, Singh KD, Bansal AK (2014) Hall current effect on viscoelastic (Walters liquid model-B) MHD oscillatory convective channel flow through a porous medium with heat radiation. Kragujev J Sci 36:19-32

23. Abdelsalam SI, Bhattic MM (2018) The study of non-Newtonian nanofluid with hall and ion slip effects on peristaltically induced motion in a non-uniform channel. R Soc Chem Adv 8:7904-7915

24. Elmaboud YA, Abdelsalam SI, Mekheimer KS (2018) Couple stress fluid flow in a rotating channel with peristalsis. J Hydrodyn 30(2):307-316. https://doi.org/10.1007/s42241-018-0037-2

25. Animasaun IL, Koriko OK, Adegbie KS, Babatunde HA, Ibraheem RO, Sandeep N, Mahanthesh B (2018) Comparative analysis between $36 \mathrm{~nm}$ and $47 \mathrm{~nm}$ alumina-water nanofluid flows in the presence of Hall effect. J Therm Anal Calorim. https://doi. org/10.1007/s10973-018-7379-4

26. Bejan A (1982) Entropy generation through heat and fluid flow. Wiley, New York

27. Adesanya SO (2014) Second law analysis for third-grade fluid with variable properties. JThermodyn 2014:452168. https://doi. org/10.1155/2014/452168

28. Adesanya SO, Makinde OD (2015) Thermodynamic analysis for a third grade fluid through a vertical channel with internal heat generation. J Hydrodyn 27(2):264-272

29. Opanuga AA, Gbadeyan JA, Agboola OO, Okagbue HI (2018) Effect of suction/injection on the entropy generation of third grade fluid with convective cooling. Defect Diffus Forum 384:465-474

30. Adesanya SO, Makinde OD (2012) Heat transfer to magnetohydrodynamic non-Newtonian couple stress pulsatile flow between two parallel porous plates. Z. Naturforschung. 67a:647-656

31. Opanuga AA, Okagbue HI, Agboola OO, Bishop SA (2018) Second law analysis of ion slip effect on MHD couple stress fluid. Int J Mech 12:96-101

32. Opanuga AA, Gbadeyan JA, lyase SA (2017) Second law analysis of hydromagnetic couple stress fluid embedded in a nonDarcian porous medium. IAENG Int J Appl Math 47(3):287-294

33. Opanuga AA, Agboola OO, Okagbue HI, Bishop SA (2018) Convection flow of MHD couple stress fluid in vertical microchannel with entropy generation. Pattern Form Stab Magnetohydrodyn. https://doi.org/10.5772/intechopen.81123

34. Rashidi MM, Bhatti MM, Abbas MA, Ali ME (2016) Entropy generation on MHD blood flow of nanofluid due to peristaltic waves. Entropy 18(4):117. https://doi.org/10.3390/e18040117

35. Ajibade AO, Jha BK, Omame A (2011) Entropy generation under the effect of suction/injection. Appl Math Model 35:4630-4646

36. Opanuga AA, Okagbue HI, Agboola OO, Imaga OF (2017) Entropy generation analysis of buoyancy effect on hydromagnetic Poiseuille flow with internal heat generation. Defect Diffus Forum 378:102-112

37. Jangili S, Adesanya SO, Falade JA, Gajjela N (2017) Entropy generation analysis for a radiative micropolar, fluid flow through a vertical channel saturated with non-Darcian porous medium. Int J Appl Comput Math 3:3759-3782. https://doi.org/10.1007/ s40819-017-0322-8

38. Srinivasacharya D, Bindu KH (2016) Entropy generation in a micropolar fluid flow through an inclined channel. Alex Eng J 55:973-982

39. Agboola OO, Opanuga AA, Okagbue HI, Bishop SA, Ogunniyi PO (2018) Analysis of Hall effects on the entropy generation of natural convection flow through a vertical microchannel. Int J Mech Eng Technol (IJMET) 9(8):712-721

40. Sohail M, Naz R, Abdelsalam SI (2019) On the onset of entropy generation for a nanofluid with thermal radiation and gyrotactic microorganisms through 3D flows. Phys Scr. https://doi. org/10.1088/1402-4896/ab3c3f

41. Zhou JK (1986) Differential transformation and its application for electrical circuits. Huarjung University Press, Wuhan (in Chinese)

42. Arikoglu A, Ozkol I (2005) Solution of boundary value problems for integro- differential equations by using differential transform method. Appl Math Comput 168:1145-1158

43. Hassan $\mathrm{HI}$ (2008) Comparison differential transformation technique with Adomian decomposition method for linear and nonlinear initial value problems. Chaos Solitons Fractals 36(1):53-65

Publisher's Note Springer Nature remains neutral with regard to jurisdictional claims in published maps and institutional affiliations. 